l problema del trattamento delle fratture del pilone tibiale è caratterizzato da alcuni fattori. Questo tipo di frattura è relativamente raro. La frattura è sempre complessa, si può dividere in meno complessa e molto complessa. Le condizioni cutanee molto facilmente sono compromesse anche in assenza di una esposizione palese e tendono a modificarsi in maniera significativa e imprevedibile nel giro di ore o giorni; una frattura di pilone tibiale a complessità maggiore andrebbe sempre considerata come una frattura esposta. L'articolarità della frattura ne richiederebbe una ricostruzione chirurgica perfetta, ma questa si scontra con problemi anatomo-patologici e tecnici. I risultati sono costantemente discutibili e presentano una curiosa contraddizione: quasi mai i risultati funzionali presentano una diretta correlazione con i risultati radiologici.

Le complicanze del trattamento non hanno mezzi termini: o non ci sono, e allora si rientra nel campo della variabilità imprevedibile dei risultati a parità di tecnica usata; oppure ci sono, e allora sono tendenzialmente gravi quando non drammatiche. I problemi per affrontare questo evento coinvolgono sovente anche l'aspetto plastico. Da qui il coinvolgimento del chirurgo plastico nello scenario.

Il trattamento perciò non richiede solo conoscenze ed esperienza tecnica notevoli, ma soprattutto la sensibilità di valutare ancora più accuratamente del normale il rapporto costi-benefici. Richiede inoltre due qualità che rientrano a pieno diritto in quelle virtù che, da Ippocrate, caratterizzano la qualità dell'azione medica: la pazienza e l'umiltà. La pazienza di aspettare ad aggredire chirurgicamente la frattura, anche a costo di polemizzare con amministratori che vorrebbero ridurre i tempi di ricovero a ogni costo; l'umiltà di ritenere che, a volte, altri colleghi possono fare meglio di noi, senza per questo sentirsi sminuiti. Ecco allora un altro elemento fondamentale del trattamento: la capacità di gestire al meglio l'attesa. Non mettere il periodo di attesa nell'algoritmo di trattamento è un errore concettuale e incide negativamente anche nell'uso della tecnica più sofisticata da parte del miglior chirurgo.

Quindi i contributi presentati in questo fascicolo rappresentano una istantanea di come si affronta il problema in situazioni reali, da cui si possono evincere gli assunti citati. Entrando nel merito tecnico, abbiamo riservato un occhio di riguardo alla fissazione esterna per alcuni motivi. Intanto un motivo di scuola. Presso il CTO di Torino la fissazione esterna ha avuto cura e amore fin dalla sua introduzione, a metà degli anni Settanta. Inoltre è una tecnica che risponde bene alle esigenze di trattamento, ma che non ha ancora i crismi della standardizzazione che ha la sintesi interna. Infine non incontra il favore né dei pazienti né dei medici, gli uni per motivi ovvi, gli altri perché la chirurgia viene vista come un atto definitivo che nel bene o nel male si conclude con l'atto operatorio. L'applicazione della fissazione esterna comporta al contrario che l'atto operatorio sia il primo passo del trattamento: all'uscita dalla sala operatoria inizia e non termina il lavoro. Da ciò l'esigenza di evidenziarne le indicazioni e di fornire il più possibile elementi di tecnica standardizzabile. La speranza è che questo

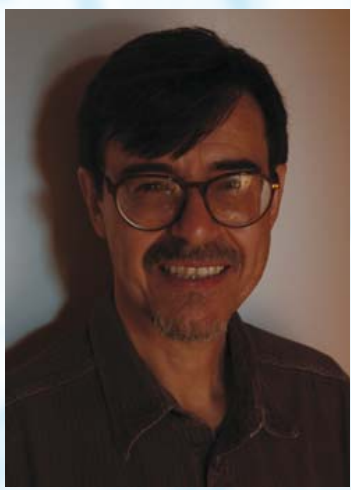

A. Biasibetti 
modesto lavoro di raccolta di esperienze introduca elementi di meditazione meritevoli poi di approfondimento. Le tecniche devono essere conosciute e applicate tutte, la discriminante essendo solo un'indicazione il più possibile corretta; la tecnica deve entrare come elemento di un algoritmo e non semplicemente come tale; le fratture complesse non devono essere considerate dal medico una disgrazia da affrontare, ma un problema risolvibile nell'ambito di una rete pensata per questo scopo. Se la rete non è istituzionalizzata, la sensibilizzazione per procedere a una sua organizzazione nelle sedi dovute può essere un onorevole impegno per chi si occupa di traumatologia. LOTODI, con l'organizzazione annuale di meeting dedicati, dimostra di comprendere che il problema di una traumatologia sempre più organizzata deve rientrare nei piani di un Sistema sanitario moderno e funzionale.

\section{A. Biasibetti}

Ospedale C.T.O.-Maria Adelaide

Torino 